\title{
Effect of BaO-CaO-SiO2 addition on dielectric and electrocaloric properties of lead-free \\ 0.2Ba(Ti0.9Sn0.1)03-0.8Ba(Zr0.18Ti0.82)03 bulk ceramics
}

\section{Zhang dandan}

Shanghai University of Engineering Science https://orcid.org/0000-0002-0736-2480

Xiuli Zhang ( $\nabla$ xlzhang@sues.edu.cn )

Shanghai University of Engineering Science

\section{XingJia LI}

Shanghai University of Engineering Science - Songjiang Campus: Shanghai University of Engineering Science

\section{Zhu LIANG}

Shanghai Institute of Ceramics Chinese Academy of Sciences

\section{Haisheng XU}

kunshan Hisense Electronics Co.,Ltd

\section{Zhaoyue LV}

East China University of Science and Technology

\section{XiaoDong SANG}

Shanghai University of Engineering Science

\section{SaiSai LI}

Shanghai University of Engineering Science

\section{Research Article}

Keywords: BZSnT20 bulk ceramics, BaO-CaO-SiO2 sintering aid, Dielectric constant, Loss tangent, Electrocaloric effect(ECE)

Posted Date: June 11th, 2021

DOl: https://doi.org/10.21203/rs.3.rs-607254/v1

License: (9) This work is licensed under a Creative Commons Attribution 4.0 International License. Read Full License 


\title{
Effect of $\mathrm{BaO}-\mathrm{CaO}-\mathrm{SiO}_{2}$ addition on dielectric and electrocaloric properties of lead-free
}

\section{$0.2 \mathrm{Ba}\left(\mathrm{Ti}_{0.9} \mathrm{Sn}_{0.1}\right) \mathrm{O}_{3}-0.8 \mathrm{Ba}\left(\mathrm{Zr}_{0.18} \mathrm{Ti}_{0.82}\right) \mathrm{O}_{3}$ bulk ceramics}

DanDan ZHANG ${ }^{a, \uparrow}$, Xiuli ZHANG ${ }^{a, *}$, XingJia LI ${ }^{a}$, Zhu LIANG ${ }^{b}$, Haisheng $\mathrm{XU}^{c, *}$, Zhaoyue $\mathrm{LV}^{d}$, XiaoDong $\mathrm{SANG}^{a}$, SaiSai LI ${ }^{a}$

${ }^{a}$ School of Mathematics and Statistics, Shanghai University of Engineering Science, Shanghai 201620, China

${ }^{b}$ Shanghai Institute of Ceramics, Chinese Academy of Sciences, Shanghai 201899, China

${ }^{c}$ Kunshan Hisense Electronics Co., Ltd., Kunshan, Jiangsu 215300, China ${ }^{d}$ Department of Physics, East China University of Science and Technology, Shanghai 200237, China

\begin{abstract}
:
In recent years, compared with traditional refrigeration technology, the electrocaloric refrigeration technology has been applied in variety of green cooling devices due to its high efficiency and environmentally friendly. Recent findings reveal that the ceramics system with additives (such as, $\mathrm{LiBiO}_{2}, \mathrm{PbO}$ ) can create large ECE under low electric field. In this work, the ternary glass $\mathrm{BaO}-\mathrm{CaO}-\mathrm{SiO}_{2}$ (BCS) was used as a sintering aid to enhance the electrocaloric (EC) response of 0.2Ba(Tio.9 $\left.\mathrm{Sn}_{0.1}\right) \mathrm{O}_{3}-0.8 \mathrm{Ba}\left(\mathrm{Zr}_{0.18} \mathrm{Ti}_{0.82}\right) \mathrm{O}_{3}(\mathrm{BZSnT} 20)$ bulk ceramics. The EC properties of BZSnT20 could be also improved via controlling sintering temperature and the content of BCS sintering aid. It was found that BZSnT20 with $0.5 \mathrm{wt} \% \mathrm{BCS}$ show the maximum EC response of $3.2 \mathrm{~K}$ under $7 \mathrm{MV} / \mathrm{m}$ with sintering temperature of $1262^{\circ} \mathrm{C}$. These results demonstrated that the ECE of BZSnT20 can be boosted by using appropriate additives, which provides an effective route to realize large ECE under low electric fields.
\end{abstract}

Keywords: BZSnT20 bulk ceramics; $\mathrm{BaO}-\mathrm{CaO}-\mathrm{SiO}_{2}$ sintering aid; Dielectric constant; Loss tangent; Electrocaloric effect(ECE) 


\section{Introduction}

New solid refrigeration technology has attracted growing attention in recent years. Much progress has been made on electrocaloric refrigeration [1], magnetic card refrigeration [2], thermoelectric refrigeration [3] and semiconductor refrigeration [4] etc. However, high cost, bulky device and the requirements of powerful magnetic field have limited the wide application of magnetic card cooling technology. Besides, the efficiency of thermoelectric refrigeration and semiconductor refrigeration technology are very low $(\leq 10 \%)$. Compared with these cooling technologies, electrocaloric refrigeration has many advantages, including high refrigeration efficiency, simple devices, low-cost and ease of miniaturization. Electrocaloric refrigeration uses electrocaloric effect (ECE) to achieve cooling effect. The term 'ECE' has been used to describe the polarization state of the electric dipole in the dielectric. By applying external electric field on EC materials, the polarization state can be changed thus resulting in isothermal entropy change $\Delta S$ and adiabatic temperature change $\Delta T[5]$. The mechanism of electrocaloric refrigeration technology determines that it is a high efficiency and environmentally friendly technology. Therefore, it has a broad prospect of application.

In recent years, in order to obtain higher electrocaloric effect at lower voltage, multi-layered thick membrane structure devices has been designed [6]. To fulfill the demand of low-temperature co-firing of the dielectric layer and the metal electrode, the sintering temperature of the electrocaloric material should be lowered [7]. Since the sintering temperature of ceramics are typically higher than the melting temperature of metal electrode materials. Fortunately, sintering aids can decrease the sintering temperature of the ceramic film, so that the metal electrode can be completely maintained after the sintering process [8]. As we all know, the introduction of appropriate sintering aids (such as, $\mathrm{B}_{2} \mathrm{O}_{3}-\mathrm{ZnO}$ [9], $\mathrm{PbO}_{-}-\mathrm{B}_{2} \mathrm{O}_{3}$ [7], $\mathrm{PbO}-\mathrm{SiO}_{2}[10], \mathrm{LiBiO}_{2}$ [7],) can not only reducing the sintering temperature, but also increasing the dielectric constant [10-12]. According to the research results from $\mathrm{Hou}$ et al.[7], via adding $\mathrm{LiBiO}_{2}$ and $\mathrm{PbO}-\mathrm{B}_{2} \mathrm{O}_{3}$ to the $\mathrm{BaZr}_{0.2} \mathrm{Ti}_{0.8} \mathrm{O}_{3}$ (BZT20) 
homogeneous double-layer thick film, a large adiabatic temperature change $\Delta T$ of $3.9 \mathrm{~K}$ was achieved under $10 \mathrm{MV} / \mathrm{m}$ electric field. Compared with $\mathrm{PbO}$ and $\mathrm{LiBiO}_{2}$, $\mathrm{BaO}-\mathrm{CaO}-\mathrm{SiO}_{2}$ (BCS) has the advantages of high chemical stability and high thermal stability. Up to now, BZT20 system is one of the most abundant EC materials in the electrocaloric refrigeration technology area, however, BCS has not been widely used as sintering aid in BZT20 system to achieve high ECE .

In this work, the influence of the BCS glass sintering aid on the sintering morphology, electrocaloric performance and dielectric properties of $0.2 \mathrm{Ba}\left(\mathrm{Ti}_{0.9} \mathrm{Sn}_{0.1}\right) \mathrm{O}_{3}-0.8 \mathrm{Ba}\left(\mathrm{Zr}_{0.18} \mathrm{Ti}_{0.82}\right) \mathrm{O}_{3}(\mathrm{BZSnT} 20)$ bulk ceramics were investigated. Besides, the mechanisms of action of BZSnT20 at different sintering temperatures and BCS sintering aid contents were also analyzed, thereby defining the optimum content and sintering temperature of BCS glass sintering aid. This work indicated that environmentally friendly electrocaloric refrigeration devices can be achieved via controlling sintering temperature and the content of BCS sintering aid in BZSnT20 system, and large ECE under low electric fields at room temperature can also be realized.

\section{Experimental section}

The BZSnT20 bulk ceramics were fabricated by the solid-state reaction method using analytical reagent grade $\mathrm{BaCO}_{3}$ (Alfa Aesar, 99.8\%), $\mathrm{TiO}_{2}$ (Alfa Aesar, 99.5\%), $\mathrm{ZrO}_{2}$ (Aladdin, 99.99\%) and $\mathrm{SnO}_{2}$, (Alfa Aesar, 99.9\%) as raw materials. The mixture of these powders were planetary milled in alcohol media with zirconia ball for 24 hours to obtain a well-dispersed mixture. After that, the mixture was calcined at $1100^{\circ} \mathrm{C}$ for 2 hours. After calcinating, the BCS sintering aids with $\mathrm{x} \mathrm{wt} \%(\mathrm{x}=0.4,0.5$, $0.6,0.7,0.8,1,1.5,2)$, additives $\left(0.5 \mathrm{~mol}^{\%} \mathrm{MnO}_{2}\right.$ and $\left.0.25 \mathrm{~mol}^{\circ} \mathrm{Nb}_{2} \mathrm{O}_{5}\right)$, and Polyvinyl alcohol (PVA) solution were added to the BZSnT20 composition. Then, the mixture was grounded in ethanol medium for 24 hours and dried. Then the mixture was pressed into pellets under the pressure of $4 \mathrm{MPa}$. Finally, the samples were sintered at $1233^{\circ} \mathrm{C}, 1245^{\circ} \mathrm{C}, 1262^{\circ} \mathrm{C}$ and $1284^{\circ} \mathrm{C}$ for 3 hours, with a heating rate of $3^{\circ} \mathrm{C} / \mathrm{min}$. For electrical characterization, the pellets were carefully polished to a thickness of $300 \mu \mathrm{m}$. Both surfaces of these polished samples were coated with silver 
as electrodes and burned at $600^{\circ} \mathrm{C}$ for 10 minutes.

The dielectric properties of BZSnT20 were characterized using a SR715 LCR meter (Stanford Research Systems). The thermal analysis of the sample was performed on the differential scanning calorimetry (DSC) (TA Q100). The heat absorb and release of the EC material were directly measured by a self-made T-type thermocouple, then the entropy variation $(\Delta S)$ was calculated according to the formula $Q=T \Delta S$.

\section{Results and discussion}

\subsection{Sintering morphology}

Figure 1 shows the morphology of BZSnT20 with different contents of sintering aid and sintering temperatures. It can be seen from figure 1 that the surface of all BZSnT20 are relatively smooth with few plaques. Interestingly, as the content of the BCS glass sintering aid is $0 \mathrm{wt} \%$, with the sintering temperature. However, with the same sintering temperature, the color of BZSnT20 gradually deepened (from yellow to brownish dark) with the contents of BCS sintering aid increases from $0.4 \mathrm{wt} \%$ to $2 \mathrm{wt} \%$. While the contents of BCS sintering aid is fixed, the color of BZSnT20 will be deepened with the increase of sintering temperature from $1233{ }^{\circ} \mathrm{C}$ to $1284{ }^{\circ} \mathrm{C}$. As a result that the addition of BCS sintering aid determine the color of BZSnT20 bulk ceramics.

\section{[Insert Fig. 1]}

\subsection{Dielectric properties}

Change of the permittivity and loss tangent (at room temperature) for ferroelectric capacitors with BCS sintering aid at different sintering temperatures are shown in Fig. 2. Interestingly, as the content of the BCS glass sintering aid in samples is $0 \mathrm{wt} \%$, the permittivity of BZSnT20 increases with the increase of sintering temperature. However, the sintering temperature did not show any significant effect on the color of samples (Fig. 1). Because the sintering temperature of BZSnT20 is increased from $1233{ }^{\circ} \mathrm{C}$ to $1284{ }^{\circ} \mathrm{C}$, which not achieving the optimal sintering temperature of BZSnT20 $\left(1400{ }^{\circ} \mathrm{C}\right)$, leading to the insufficient sintering of BZSnT20 
[13]. The insufficient sintering of BZSnT20 leads to a light color and a low dielectric constant.

With increasing of the content for BCS glass sintering aid in samples, the permittivity of BZSnT20 shows a tendency of increasing first and then decreasing. And the color of BZSnT20 is gradually deepened (from yellow to brownish)(Fig.1). As the content for the BCS glass sintering aid in samples is up to $0.5 \mathrm{wt} \%$, the dielectric constant shows a peak, which is a 2.5-fold increase compared to BZSnT20 of no BCS glass sintering aid. The loss tangent as a function of BCS glass content is shown in Figure 2(b). It is worth mentioning that, at the same content $(0.4 \mathrm{wt} \%)$ of BCS glass sintering aid in samples, the loss tangent of the sample also show a turning piont for decreasing first and then increasing. Then with the increase of BCS sintering aid, the loss tangent keeps a relatively low level. Further, it is found that the $\mathrm{Ca}^{2+}$ and $\mathrm{Si}^{4+}$ in BCS will increase with the content of increasing of BCS sintering aid. It is mention that $\mathrm{Ca}^{2+}$ enters the $\mathrm{A}$ site of the perovskite structure, meanwhile $\mathrm{Si}^{4+}$ enters the $\mathrm{B}$ site of the perovskite structure, so that the substitution of $\mathrm{Ba}^{2+}$ with $\mathrm{Ca}^{2+}$, the substitution of $\mathrm{Zr}^{4+}$ and $\mathrm{Ti}^{4+}$ with $\mathrm{Si}^{4+}$. The occurrence of replacement will induce the lattice volume shrinkage of BZSnT20 bulk ceramics, which in turn induce the lattice deformation, internal stress, the increase of permittivity and the deep of colors [14-19] .At the same time, the BCS liquid phase can get into the grain boundaries of BZSnT20 bulk ceramics during the sintering process, which reduces the defects in the ceramics grain, along with loss tangent decreasing further [20].

However, with the excessive addition of BCS glass sintering aid (1,1.5 and $2 \mathrm{wt} \%$ ), the dielectric constant of BZSnT20 is gradually decrease and the color is gradually darkens(Fig. 1). Because $\mathrm{Ca}^{2+}$ and $\mathrm{Si}^{4+}$ can access to the band gap position of the crystal, forming more impurity phases. It can be seen that the ionic radius of $\mathrm{Ca}^{2+}(0.1 \mathrm{~nm})$ is larger than the ionic radius of $\mathrm{Ti}^{4+}(0.065 \mathrm{~nm})$ and $\mathrm{Zr}^{4+}(0.072 \mathrm{~nm})$, so that the $\mathrm{Ca}^{2+}$ can be restricted by the solid solubility, then some excess $\mathrm{Ca}^{2+}$ starts to entry the $\mathrm{B}$ site of the perovskite structure. The substitution of $\mathrm{B}$ site ion with $\mathrm{Ca}^{2+}$, as a result, the spontaneous polarization of the system and the permittivity will decrease with the lattice volume expansion $[21,22]$. It is all known that $\mathrm{Zr}^{4+}$ or $\mathrm{Ti}^{4+}$ is partially 
substituted by $\mathrm{Ca}^{2+}$, which is accompanied by the transition of electrons between ions, producing a part of oxygen vacancies [23]. The presence of oxygen vacancies can reduce the space of oxygen octahedrons, resulting in the decrease of the permittivity of bulk ceramics. The BZSnT20 bulk ceramics, sintered with the $0.5 \mathrm{wt} \%$ BCS exhibit the permittivity maximum up to 11000 at $1284^{\circ} \mathrm{C}$ sintering temperature. But BZSnT20 shows a relatively small slope at $1284^{\circ} \mathrm{C}$ sintering temperature compared to the samples at $1262^{\circ} \mathrm{C}$ sintering temperature, and the increase of the permittivity is rather limited from $1262^{\circ} \mathrm{C}$ to $1284^{\circ} \mathrm{C}$. Meanwhile, it can also be seen (Fig. 2b) that the loss tangent of all sample are relatively low at the sintering temperature of $1262^{\circ} \mathrm{C}$. The combination of relatively high permittivity and relatively low loss tangent in BZSnT20 bulk ceramics is highly desirable for strengthening the electrocaloric effect. Therefore, the electrocaloric effect of BZSnT20 bulk ceramics at the sintering temperature of $1262^{\circ} \mathrm{C}$ are discussed below.

\section{[Insert Fig. 2]}

\subsection{Electrocaloric performance}

According to the formula

$$
\frac{d T}{\mathrm{~d} E}=\frac{a_{0} \varepsilon_{0} \varepsilon_{r} T P}{c_{E}}
$$

In an EC strength equation, $a_{0}, \mathrm{~T}$ and $\mathrm{P}$ are Phenomenological coefficients, adiabatic temperature and polarization strength, respectively; $\varepsilon_{\mathrm{r}}$ and $\varepsilon 0$ are the dielectric constant of materials and vacuum. $\left(\frac{\mathrm{d} T}{\mathrm{~d} E}\right)$ can be calculated using Eq. (1), further confirming that it is influenced by the dielectric constant of materials [24]. Thus, in order to design EC strength materials with a giant ECE, a large dielectric constant is desired.

Figure 3 displays the directly recorded EC signal of BZSnT20 bulk ceramics (300um) under $5 \mathrm{MV} / \mathrm{m}$ at room temperature. Applying an electric field on the BZSnT20 bulk ceramics, the dipoles of the ceramic, it is the change from a disordered arrangement to an ordered arrangement leading to an exothermic process, which is 
one of the source of the ECE. As the applied electric is removed, the endothermic peak is obtained around room temperature by the dipole changes from order to disorder, displayed in the peak in Fig. 3(a). In general, the heat Q can be estimated by the integral area of the exothermic and endothermic peak. The isotherm entropy change $\Delta S$ is estimated from $Q=T \Delta S$. The adiabatic temperature change $\Delta T$ is calculated according to $Q=c_{E} \Delta T$, where $c_{E}$ is the specific heat capacity of the ceramic at room temperature. Thus, the values obtained from exothermic and endothermic peaks are nearly the same during both EC heating and cooling process [25].

As shown in Fig.3(b), electric field-induced ECE increases linearly with the electric field for all these BZSnT20 bulk ceramics. With increasing of the content for BCS glass sintering aid in samples, from $0 \mathrm{wt} \%$ to $1.0 \mathrm{wt} \%$, the ECE of BZSnT20 bulk ceramics presents a trend of first increasing and then decreasing. Hou et al. reported that the entropy change reaches $4.8 \mathrm{~J} \cdot \mathrm{Kg}^{-1} \cdot \mathrm{K}^{-1}$ under an electric field of $10 \mathrm{MV} / \mathrm{m}$ [26]. It is noted that under an low electric field of $7 \mathrm{MV} / \mathrm{m}$, a large $\mathrm{ECE}(\Delta T=3.2 \mathrm{~K}$, $\left.\Delta S=4.8 \mathrm{~J} \cdot \mathrm{Kg}^{-1} \cdot \mathrm{K}^{-1}\right)$ is achieved using $0.5 \mathrm{wt} \% \mathrm{BCS}$ addition in this work, which data are summarized in Table 1. To gain more detailed information on enhancement ECE on $\mathrm{BaTiO}_{3}$ ceramics system, we note that the modification of $\mathrm{BaTiO}_{3}$ ceramics system by the elements of $\mathrm{Zr}$ and $\mathrm{Sn}$, may be formed the four phases (paraelectric cubic, ferroelectric tetragonal, orthogonal and rhombohedral), and converged into a multi-phase point transition (critical point ICP) [27-28 ].The fact that multi-phase coexistence can decrease the electric field required and thus enhance the ECE [29]. In addition, the $\mathrm{Ca}^{2+}$ and $\mathrm{Si}^{4+}$ in $\mathrm{BCS}$ glass sintering aids could shift to the samples matrix, then partially replace A and B sites in perovskite structure, so that improving a huge ECE substantially at room temperature. However, with the excessive addition of BCS glass sintering aid (1wt.\%), $\mathrm{Ca}^{2+}$ and $\mathrm{Si}^{4+}$ can enter the band gap position of the crystal, forming more impurity phases, and the ECE may be weakened partly. It is worth noting that the BZSnT20 with $1.0 \mathrm{wt} . \%$ BCS sintering aid is easy to be ruptured 
even at a low electric field of $5 \mathrm{MV} / \mathrm{m}$. This might be mainly caused by the field-driven strain of the dielectric layers and structural defects in BCS samples with $1.0 \mathrm{wt} \%$ BCS sintering aid. More importantly, it indicates that a larger EC response could exist in BCS-added BZSnT20 bulk ceramics with $0.5 \mathrm{wt} \% \mathrm{BCS}$, so that ECE and breakdown electric field could be modified through employing tiny additives.

\section{[Insert Fig. 3]}

The ambient temperature dependence of ECE for BZSnT20 using BCS as additives is shown in Figure 4. With the increase of ambient temperature, from $0{ }^{\circ} \mathrm{C}$ to $60{ }^{\circ} \mathrm{C}$, the ECE gradually increase and finally tend to a constant. This was primarily attributed to the different orientations of the dipoles in BZSnT20 at various ambient temperatures, resulting in the change of ECE [30]. Therefore, the diagram is divided in two areas that it is separated the low temperature region (I) and the higher temperature region (II). In region I, the ECE increases with the ambient temperature increasing from $0{ }^{\circ} \mathrm{C}$ to $20{ }^{\circ} \mathrm{C}$. In region II, as the ambient temperature increases from $20{ }^{\circ} \mathrm{C}$ to $60{ }^{\circ} \mathrm{C}$, the ECE keeps basically at the same level. Therefore, the electrocaloric performance of BZSnT20 with $0.5 \%$ BCS is relatively stable near room temperature, and it can produce a huge ECE over a wide operating temperature (from $20^{\circ} \mathrm{C}$ to $\left.60^{\circ} \mathrm{C}\right)$, even under a relatively lower electric field $(5 \mathrm{MV} / \mathrm{m})$. Table 1 lists the ECE of this work and previously reported in the literature. The ECE data from bulk ceramics, bilayer thick films and thick films are also included, and it can be effectively improved by using addition, such as, $\mathrm{BaO}-\mathrm{CaO}-\mathrm{SiO}_{2}, \mathrm{LiBiO}_{2}, \mathrm{PbO}+\mathrm{B}_{2} \mathrm{O}_{3}$, and $\mathrm{CuO}$. Comparing the electric field, ambient temperature and ECE, the BZSnT20 bulk ceramics with $\mathrm{BaO}-\mathrm{CaO}-\mathrm{SiO}_{2}$ addition can achieve larger ECE at lower electric field $(7 \mathrm{MV} / \mathrm{m})$. Therefore, The results of the present study suggested that the ECE could be effectively enhanced by employing small amount of appropriate additives.

\section{[Insert Fig. 4 ]}

[Insert Table. 1]

\section{Conclusion}


In summary, high efficiency ECE as an environmentally friendly refrigeration technology has been studied via modifying the BZSnT20 system with BCS sintering aid. The ECE of the BZSnT20 system has shown a clear correlation with different contents of BCS sintering aid, presented by sintering morphology, dielectric properties and electrocaloric properties. As a device of refrigeration technology, the BZSnT20 ferroelectric capacitors exhibits a large ECE of 3.2K. The results show that a large ECE under low electric field can be realized by optimizing the microstructure configuration of BZSnT20 controlled by BCS-added additives and sintering temperature, providing a facile efficient approach to design advanced EC.

\section{Acknowledgements}

The project was supported by the National Natural Science Foundation of China (Grant No. 51503121), the Open Project of the State Key Laboratory of Infrared Physics, Chinese Academy of Sciences (Grant No. IIMDKFJJ-20-11)and the Talent Program of Shanghai University of Engineering Science (Grant No. 2018RC54).

\section{References}

[1] Scott J F. Electrocaloric materials. J Annual Review of Materials Research 2011, 41: $229-240$.

[2] Alzate-Cardona J D, Salcedo-Gallo J S, Rodríguez-Patiño D F, et al. Unveiling a scaling and universal behavior for the magnetocaloric effect in cubic crystal structures: a Monte Carlo simulation. J Scientific reports 2019, 9: 1-7.

[3] Wang N, Chen J, Zhang K, et al. Equivalent model optimization with cyclic correction approximation method considering parasitic effect for thermoelectric coolers. J Scientific reports 2017, 7: 1-10.

[4] Yangui A, Bescond M, Yan T, et al. Evaporative electron cooling in asymmetric double barrier semiconductor heterostructures. J Nature communications 2019, 10: $1-7$.

[5] Lu SG, Zhang Q. Lctrarge electrocaloric effect in relaxor ferroelectrics. J Journal of Advanced Dieleics 2012, 2: 1230011. 
[6] Nomura T. Recent progress of multilayer ceramic capacitors[C]//Third International Conference on Thin Film Physics and Applications. International Society for Optics and Photonics 1998, 3175: 262-268.

[7] Hou Y, Yang L, Zhao X, et al. Sintering aids modified electrocaloric response in BaZr0.2Ti0.8 $\mathrm{O}_{3}$ bilayer films. J Journal of Alloys and Compounds 2017, 724: 8-13.

[8] Hayashi T, Inoue T, Akiyama Y. Low-temperature sintering and properties of $(\mathrm{Pb}, \mathrm{Ba}, \mathrm{Sr})(\mathrm{Zr}, \mathrm{Ti}, \mathrm{Sb}) \mathrm{O}_{3}$ piezoelectric ceramics using sintering aids. J Japanese Journal of Applied Physics 1999, 38: 5549.

[9] Wang SB, Dai GZ, Yao YB, et al. Direct and indirect measurement of large electrocaloric effect in $\mathrm{B}_{2} \mathrm{O}_{3}-\mathrm{ZnO}$ glass modified $\mathrm{Ba}_{0.65} \mathrm{Sr}_{0.35} \mathrm{TiO}_{3}$ bulk ceramics. $J$ Scripta Materialia 2021, 193: 59-63.

[10]Kuromitsu Y, Wang S F, Yoshikawa S, et al. Interaction between barium titanate and binary glasses. J Journal of the American Ceramic Society 1994, 77: 493-498.

[11]Chowdary K R, Subbarao E C. Liquid phase sintered BaTiO3. J Ferroelectrics 1981, 37: 689-692.

[12]Burn I. Flux-sintered $\mathrm{BaTiO}_{3}$ dielectrics. J Journal of Materials Science 1982, 17 : 1398-1408.

[13] Xu Y, Zhang $\mathrm{K}, \mathrm{Fu} \mathrm{L}$, et al. Effect of $\mathrm{MgO}$ addition on sintering temperature, crystal structure, dielectric and ferroelectric properties of lead-free BZT ceramics. J Journal of Materials Science: Materials in Electronics 2019, 30: 7582-7589.

[14]Niu X, Jian XD, Chen XY, et al. Enhanced electrocaloric effect at room temperature in $\mathrm{Mn}^{2+}$ doped lead-free $(\mathrm{BaSr}) \mathrm{TiO}_{3}$ ceramics via a direct measurement. J Journal of Advanced Ceramics 2021, 10:482-492.

[15]Du H, Liu D, Tang F, et al. Microstructure, piezoelectric, and ferroelectric properties of $\mathrm{Bi}_{2} \mathrm{O}_{3}$-added $\left(\mathrm{K}_{0.5} \mathrm{Na}_{0.5}\right) \mathrm{NbO}_{3}$ lead-free ceramics. $J$ Journal of the American Ceramic Society 2007, 90: 2824-2829.

[16]Zhang W, Eitel R E. Low-temperature sintering and properties of 0.98PZT-0.02SKN ceramics with $\mathrm{LiBiO}_{2}$ and $\mathrm{CuO}$ addition. J Journal of the American Ceramic Society 2011, 94: 3386-3390. 
[17]Hayashi T, Hasegawa T, Akiyama Y. Enhancement of piezoelectric properties of low-temperature-fabricated $\mathrm{Pb}\left(\mathrm{Mg}_{1 / 3} \mathrm{Nb}_{2 / 3}\right) \mathrm{O}_{3}-\mathrm{PbZrO}_{3}-\mathrm{PbTiO}_{3}$ ceramics with $\mathrm{LiBiO}_{2}$ sintering aid by post-annealing process. J Japanese Journal of Applied Physics 2004, 43: 6683.

[18]Im I H, Chung K H. Dielectric and Piezoelectric Properties of Lead-Free 0.5Ba $\left(\mathrm{Zr}_{0.2} \mathrm{Ti}_{0.8}\right) \mathrm{O}_{3}-0.5\left(\mathrm{Ba}_{0.7} \mathrm{Ca}_{0.3}\right) \mathrm{TiO}_{3}$ Piezoelectric Ceramics with Glass Additive. $J$ Journal of Nanoscience and Nanotechnology 2014, 14: 8920-8924.

[19]Zheng P, Zhang JL, Tan YQ, et al. Grain-size effects on dielectric and piezoelectric properties of poled $\mathrm{BaTiO}_{3}$ ceramics. J Acta Materialia 2012, 60: 5022-5030.

[20]Kang W, Zheng Z, Li Y, et al. Effect of doping $\mathrm{CaO}$ on dielectric properties of $\mathrm{BaZr}_{0.05} \mathrm{Ti}_{0.95} \mathrm{O}_{3}$ ceramics. J Digest Journal of Nanomaterials And Biostructures 2018, 13: 713-719.

[21]Sun Z, Li L, Zheng $\mathrm{H}$, et al. Effects of sintering temperature on the microstructure and dielectric properties of $\mathrm{BaZr}_{0.2} \mathrm{Ti}_{0.8} \mathrm{O}_{3}$ ceramics. J Ceramics International 2015, 41: 12158-12163.

[22]Hayati R, Razavian M A. Dielectric and Mechanical Properties of BZT-xBCT Piezoceramics Modified by $\mathrm{Nano} \mathrm{SiO}_{2}$ Additive. J Advanced Ceramics Progress 2020, 6: 24-29.

[23] Yao S, Zhou S, Buerger D, et al. Decisive role of oxygen vacancy in ferroelectric vs. ferromagnetic Mn-doped BaTiO3 thin films. J Journal of Applied Physics 2011, 109:155.

[24]Li SG, Li DD, Li XW, et al. Influence of electric field on the phenomenological coefficient and electrocaloric strength in ferroelectrics. J Acta Physica Sinica 2020, 69.(in Chinese)

[25]Bai Y, Han X, Zheng XC, et al. Both high reliability and giant electrocaloric strength in $\mathrm{BaTiO}_{3}$ ceramics. J Scientific reports 2013, 3: 1-5.

[26] Hou Y, Li J, Ding J, et al. Giant electrocaloric response in compositional manipulated $\mathrm{BaTiO}_{3}$ relaxor-ferroelectric system. J Journal of Applied Physics 2020, 127: 064103. 
[27]Hou Y, Yang L, Qian X, et al. Electrocaloric response near room temperature in Zr-and Sn-doped $\mathrm{BaTiO}_{3}$ systems. J Philosophical Transactions of the Royal Society A: Mathematical, Physical and Engineering Sciences 2016, 374: 20160055.

[28]Upadhyay SK, Fatima I, Reddy VR. Study of electrocaloric effect in Ca and Sn co-doped $\mathrm{BaTiO}_{3}$ ceramics. J Mater Res Express 2017, 4: 046303.

[29]Hou Y, Yang L, Qian X, et al. Enhanced electrocaloric effect in composition gradient bilayer thick films. J Applied Physics Letters 2016, 108: 133501.

[30]Pan R, Pan Y, Chen Y, et al. Influence of ferroelectricity and dipole turning onelectrocaloric effect. J Scientia Sinica Physica, Mechanica \& Astronomica 2019, 50: 037701.(in Chinese)

[31]Qian XS, Ye HJ, Zhang YT, et al. Giant electrocaloric response over a broad temperature range in modified $\mathrm{BaTiO}_{3}$ ceramics. $J$ Advanced Functional Materials 2014, 24: 1300-1305.

[32]Zhang N, Zheng T, Zhao C, et al. Enhanced electrocaloric effect in compositional driven potassium sodium niobate-based relaxor ferroelectrics. J Journal of Materials Research 2021: 1-11. 
Fig. 1 The sintered morphology of BZSnT20 ferroelectric capacitors fabricated with different BCS glass sintering aid contents at different sintering temperature.

Fig. 2 (a) The BCS glass content dependence of dielectric constant at various sintering temperatures. (b) The BCS glass content dependence of loss tangent at various sintering temperatures.

Fig. 3 (a)The directly measured EC signal for the sample as the electric field was turned on and off, respectively. The date is measured at an applied field of $5 \mathrm{MV} / \mathrm{m}$ and room temperature. (b) Electric field dependence of EC-induced $\Delta T$ and $\Delta S$ for BZSnT20 ferroelectric capacitors with different content sintering aids at sintering temperature of $1262^{\circ} \mathrm{C}$ and room temperature.

Fig. $4 \Delta T$ and $\Delta S$ as functions of ambient temperature for BZSnT20 ferroelectric capacitors with $0.5 \% \mathrm{BCS}$ sintering aids under electric fields of $5 \mathrm{MV} / \mathrm{m}$ and $1262^{\circ} \mathrm{C}$ sintering temperature.

Table 1 Comparison of EC properties of the BZSnT20 ferroelectric capacitors developed here with those in the literatures. 


\section{Figures}

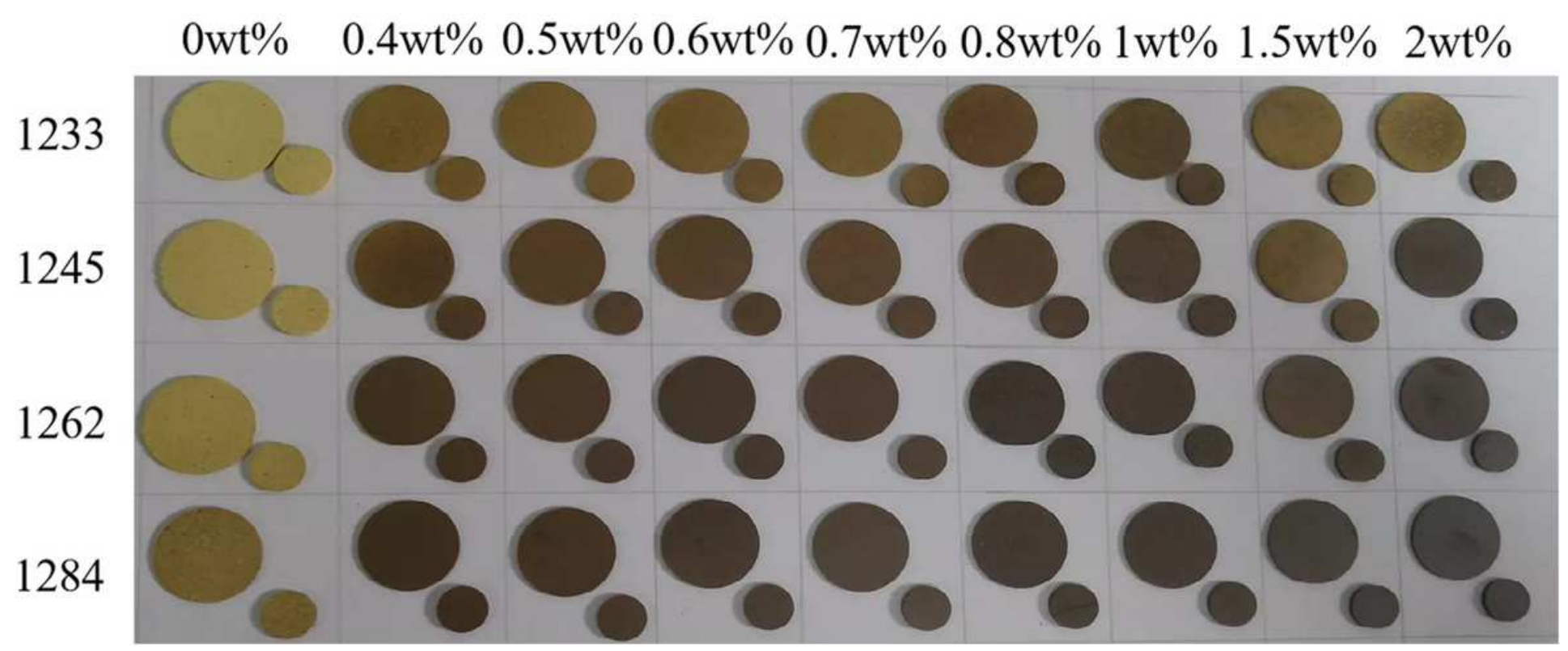

Figure 1

The sintered morphology of BZSnT20 ferroelectric capacitors fabricated with different BCS glass sintering aid contents at different sintering temperature.
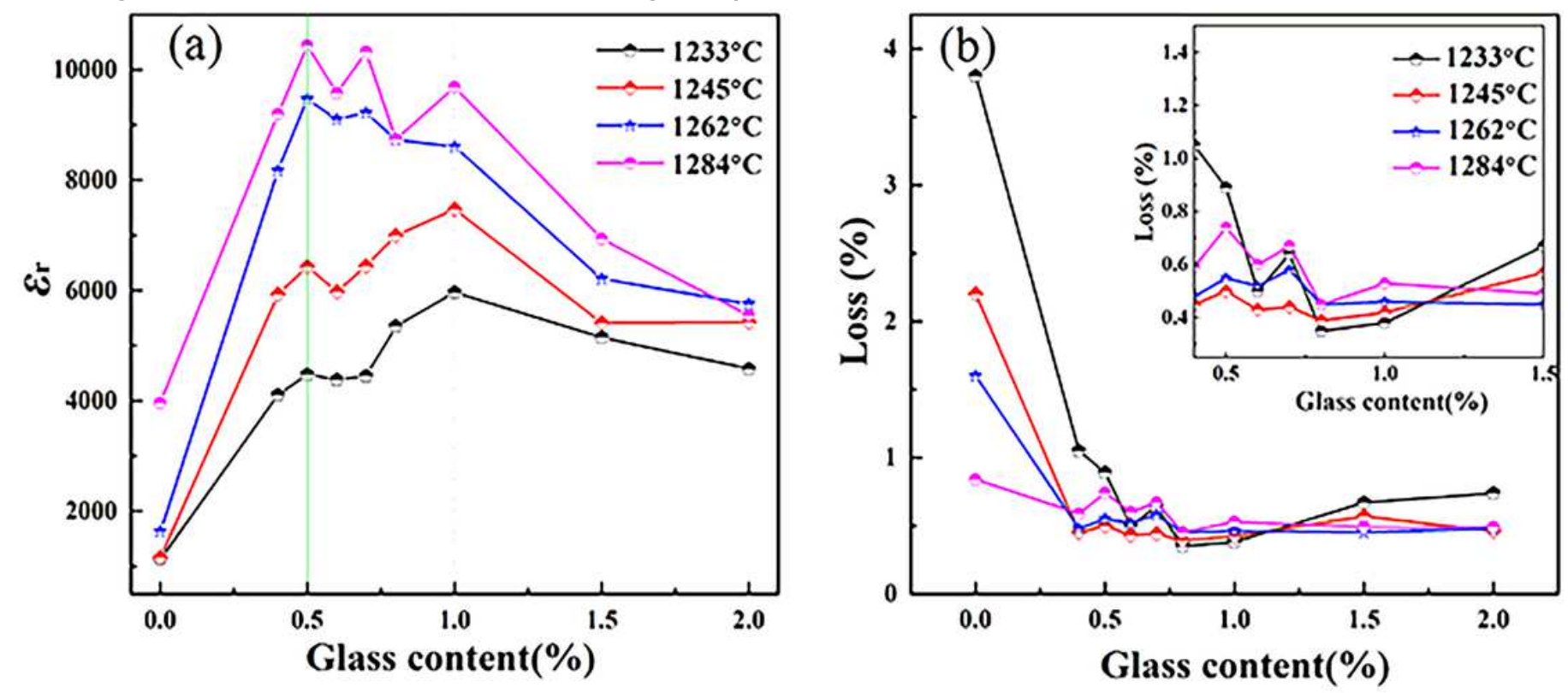

Figure 2

(a) The BCS glass content dependence of dielectric constant at various sintering temperatures. (b) The BCS glass content dependence of loss tangent at various sintering temperatures. 

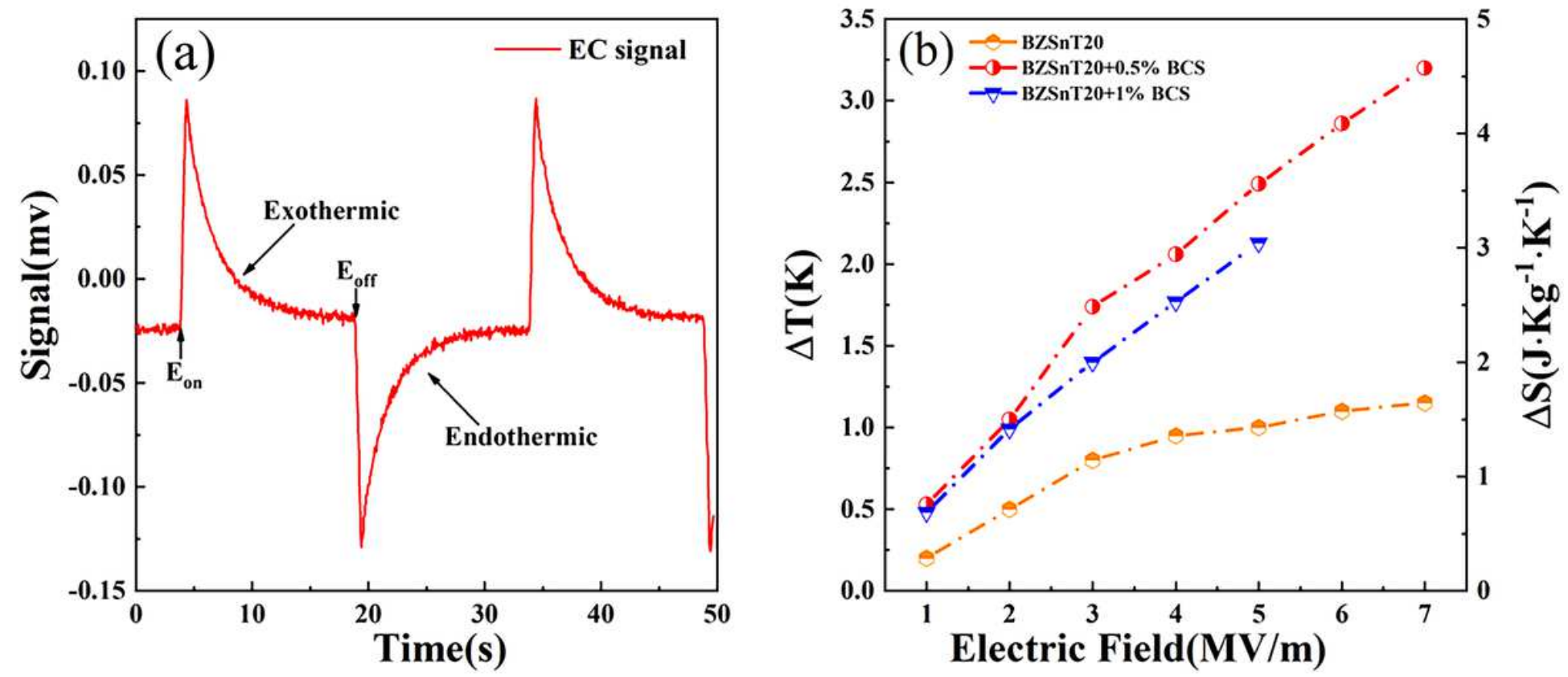

Figure 3

(a)The directly measured EC signal for the sample as the electric field was turned on and off, respectively. The date is measured at an applied field of $5 \mathrm{MV} / \mathrm{m}$ and room temperature. (b) Electric field dependence of EC-induced and for BZSnT20 ferroelectric capacitors with different content sintering aids at sintering temperature of $1262^{\circ} \mathrm{C}$ and room temperature. 


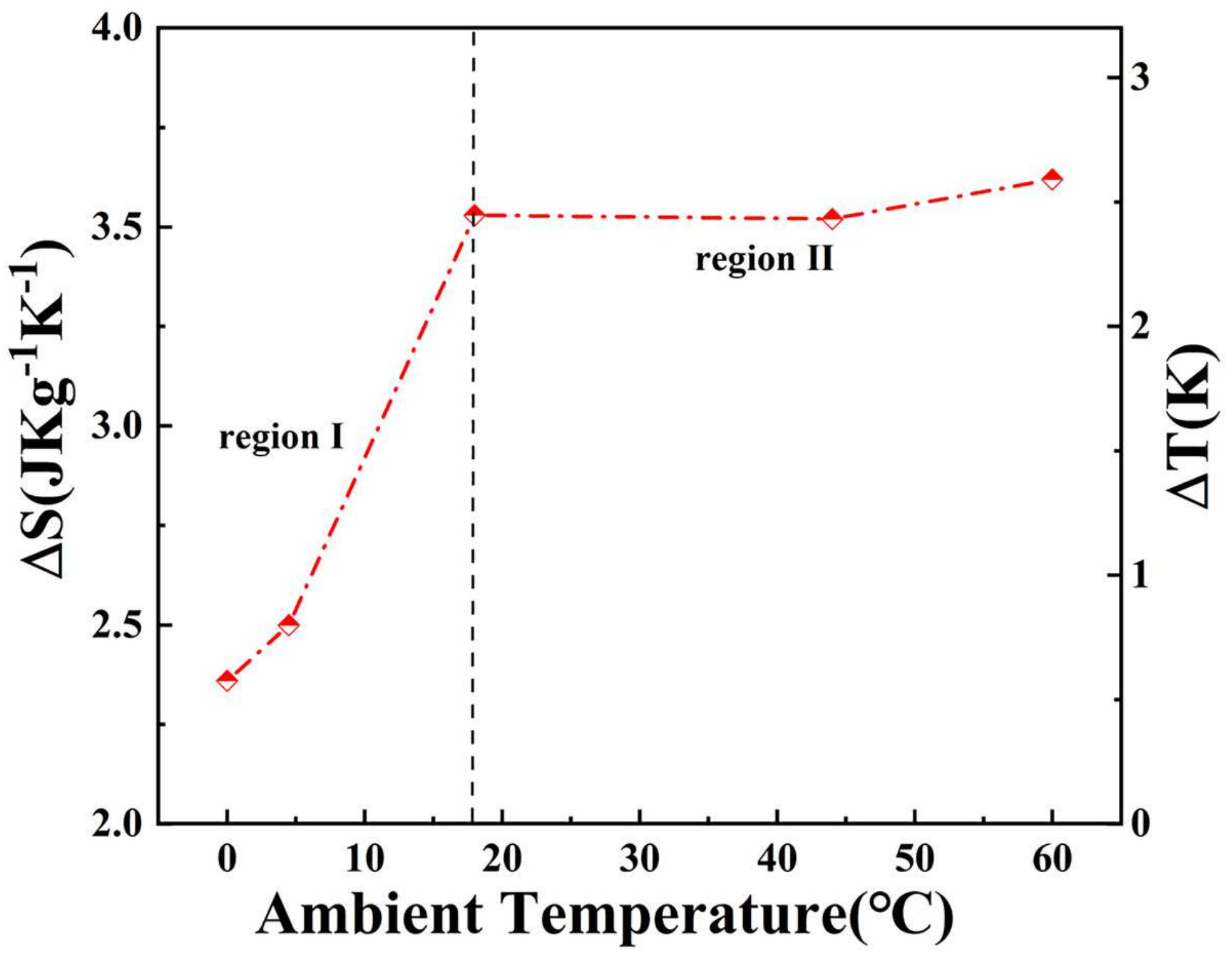

Figure 4

and as functions of ambient temperature for BZSnT20 ferroelectric capacitors with $0.5 \%$ BCS sintering aids under electric fields of $5 \mathrm{MV} / \mathrm{m}$ and $1262 \bigotimes$ sintering temperature. 